\title{
Is there a role for hyberbaric oxygen as primary treatment for grade IV radiation-induced haemorrhagic cystitis? a prospective pilot-feasibility study and review of literature
}

Athanasios Dellis ${ }^{1}$, Charalambos Deliveliotis², Vasileios Kalentzos ${ }^{3}$, Pavlos Vavasis ${ }^{3}$, Andreas Skolarikos ${ }^{2}$

${ }^{1}$ 2nd Surgical Department, University of Athens, Aretaieion Hospital; ${ }^{2} 2$ nd Urologic Department, University of Athens, Sismanoglio General Hospital and ${ }^{3}$ Diving and Hyperbaric Oxygen Department, Naval and Veterans Hospital, Athens, Greece

ABSTRACT

Purpose: To examine the safety and efficacy of hyperbaric oxygen as the primary treatment for Grade IV radiation-induced haemorrhagic cystitis.

Materials and Methods: Hyperbaric oxygen was prospectively applied as a primary treatment option in 11 patients with Grade IV radiation cystitis. Primary endpoint was the incidence of complete and partial response to treatment. Secondary endpoints included the duration of response, the correlation of treatment success-rate to the interval between the onset of haematuria and initiation of therapy, blood transfusion need and total radiation dose, the number of sessions to success, the avoidance of surgery and the overall survival.

Results: All patients completed therapy without complications for a mean follow-up of 17.82 months (range 3 to 34). Mean number of sessions needed was 32.8 (range 27 to 44). Complete and partial response rate was $81.8 \%$ and $18.2 \%$, respectively. However, in three patients the first treatment session was not either sufficient or durable giving a $72.7 \%$ rate of durable effect. Interestingly, all 9 patients with complete response received therapy within 6 months of the haematuria onset compared to the two patients with partial response who received therapy at 8 and 10 months from the haematuria onset, respectively $(p=0.018)$. The need for blood transfusion $(p=0.491)$ and the total radiation dose ( $p=0.259)$ were not correlated to success-rate. One patient needed cystectomy, while all patients were alive at the end of follow-up.

Conclusions: Early primary use of hyperbaric oxygen to treat radiation-induced grade IV cystitis is an effective and safe treatment option.
ARTICLE INFO

Key words:

Hyperbaric Oxygenation;

Radiation; Cystitis

Int Braz J Urol. 2014; 40: 296-305

Submitted for publication:

November 28, 2012

Accepted after revision:

September 17, 2013

\section{INTRODUCTION}

The inhalation of oxygen in high percentages and under high pressure has been extensively applied to the prevention and treatment of complications after radiation therapy (1). Recently, the European Society for Therapeutic Radiotherapy and
Oncology and the European Committee for Hyberbaric Medicine underlined the indications of hyberbaric oxygen therapy (HBO) in the treatment of radio-induced lesions in normal tissue (1), including the prevention of osteoradionecrosis after dental extraction, the treatment of mandibular osteoradionecrosis in combination with surgery and the treatment of 
haemorrhagic cystitis resistant to conventional treatments. All studies published on HBO therapy for radiation cystitis are not randomized or controlled. The majority constitutes retrospective reviews and case series (2-13), with only one study being prospective in nature (14). In all these studies HBO was used as a secondary treatment option. However, experimental (15-17) and clinical studies (2-13) indicate that HBO may correct the underlying pathophysiology of radiocystitis, leading to permanent cure. We present the first prospective series on hyberbaric oxygen therapy of radiation cystitis in patients who have not received any previous treatment.

\section{MATERIALS AND METHODS}

\section{Inclusion/Exclusion criteria}

Since September 2007 we have prospectively enrolled 11 patients with grade IV post-radiation haematuria morbidity, according to the Radiation Therapy Oncology Group (RTOG) and the European Organization for Research and Treatment of Cancer (EORTC) acute and late radiation morbidity scoring criteria for radiation-induced haemorrhagic cystitis (Table-1) (18). As a consequence, all patients suffered from severe haemorrhagic cystitis with haematuria requiring transfusion. Furthermore, we have prospectively classified our cases into two groups accor- ding to transfusion needs prior to HBO treatment; the first group included patients who required 1-6 units of packed red cells and the second included patients who required more than 6 units of packed red cells, respectively. All patients should have not had any prior treatment of the radiation cystitis, apart from bladder irrigation. Patients with severe emphysema or other severe chronic obstructed airway disease, a history of spontaneous pneumothorax, a history of tympanic membrane spontaneous perforation or otological reconstruction were excluded from the current study. Moreover, patients with active viral infection, history of treatment with cisplatin or doxorubicin, active bladder malignancy and uncorrected bleeding disorders were not considered eligible for our study.

\section{Evaluation and Treatment}

On admission to hospital all patients underwent full blood count and complete clotting and biochemistry profile measurements. Urine samples for common urinalysis, culture and cytology were also sent to laboratory. Computed tomography (CT) or Magnetic Resonance Imaging (MRI) of the abdomen was scheduled for all patients to access the status of the underlying malignancy and to exclude other bladder pathology. All patients underwent cystoscopy under anesthesia

Table 1 - Classification of haematuria events for both acute and late radiation morbidity scoring criteria for radiation-induced haemorrhagic cystitis.

\begin{tabular}{|c|c|c|}
\hline & \multicolumn{2}{|c|}{ Radiation morbidity } \\
\hline $\begin{array}{l}\text { Haematuria } \\
\text { morbidity }\end{array}$ & $\begin{array}{c}\text { Acute }^{*} \\
\left(\mathrm{RTOG}^{\star \star}\right)\end{array}$ & $\begin{array}{c}\text { Late }^{\star} \\
(\text { RTOG/EORTC***) }\end{array}$ \\
\hline Grade I & NA & Minor telangiectasia (microscopic haematuria) \\
\hline Grade II & NA & Generalized telangiectasia (macroscopic haematuria) \\
\hline Grade III & Gross haematuria with or without clot passage & $\begin{array}{l}\text { Severe generalized telangiectasia (frequent } \\
\text { macroscopic haematuria) }\end{array}$ \\
\hline Grade IV & Haematuria requiring transfusion & Severe haemorrhagic cystitis \\
\hline Grade V & Death from uncontrolled haematuria & Death from uncontrolled haematuria \\
\hline
\end{tabular}


and bladder biopsies were taken by one surgeon to confirm histological changes consistent with radiation cystitis and to exclude bladder malignancy.

Based on the results of previous studies (214), all patients were initially scheduled to receive 30 HBO sessions in a walk-in multiplace hyberbaric chamber with intend to increase them up to 45 until the haematuria resolved. Patient would receive $100 \%$ oxygen at a 1.8 atmospheres absolute pressure per session for 90 minutes per day, five days a week- Monday to Friday, schedule which is compatible with the definition of HBO therapy according to the Undersea and Hyberbaric Medicine Society (19). When complete response to HBO was reached the treatment was ceased. In case of improvement only or relapse of haematuria during follow-up, therapy was planned to re-initiate under the same schedule. In case no benefit was gained from the initial treatment, more than 45 treatments needed, severe complications occurred, or the patient declined further therapy, HBO was considered as a failure and the plan was to refer the patient for having another treatment, either conservative or surgical. During treatment all patients were closely monitored. Serum glucose measurements were planned as needed in diabetic patients to exclude hypoglycemia.

Within 4 weeks of treatment completion, all patients underwent cystoscopy under anesthesia and bladder biopsy by the same surgeon to confirm treatment result and/or to compare with the pre-treatment status.

\section{Study endpoints}

Primary endpoint was the success rate measured by the incidence of complete and partial response to treatment. Complete response was defined as the complete cessation of bleeding and the lack of need for transfusion in combination with the disappearance of endoscopic findings and concomitant normal bladder findings in repeat biopsies. Partial response was defined as a decrease in the grade of RTOG/EORTC scoring criteria mainly the existence of microscopic haematuria or the persistence of mild macroscopic haematuria not requiring transfusion or other urgent treatment. Secondary endpoints included the duration of $\mathrm{HBO}$ response without need for further treatment, the correlation of the time interval between initiation of HBO therapy and the onset of haematuria to the success rate of HBO therapy, the correlation of blood transfusion need to HBO success rate, the correlation of total Gys received to the HBO success rate, the number of sessions needed to achieve success, the avoidance of surgery and the overall survival.

\section{Statistical analysis}

Descriptive statistics and comparisons were made using the SPSS 12 statistical package with $\mathrm{p}<0.05$ being significant. Statistical analysis was performed using the chi-square and t test, as appropriate.

\section{RESULTS}

Since September 2007 ten men and one woman were enrolled in our study. Mean patient age was 71.7 years (range 56 to 82). Indications for radiation therapy included prostate cancer for all male patients and bladder cancer for the female patient. Mean radiation dose was 74.45 Gy (range 66 to 80). Mean interval between completion of radiation therapy and onset of haematuria was 9.8 months (range 4 to 14). Mean interval between completion of radiation therapy and the onset of HBO therapy was 13.11 months (range 6 to 22 months). Mean transfusion need prior to and during the treatment was 8.18 red blood cells packs (range 3 to 16). More specifically 4 patients have been transfused with $\leq$ 6 units and 7 patients with $>6$ units.

Pretreatment patient evaluation revealed no bladder infection or tumor. Bladder biopsy revealed diffuse mucosal edema, vascular telangiectasia, submucosal haemmorhage, interstitial fibrosis and fibrosis of smooth muscle, histological findings consistent with post-radiation cystitis. Severe ischemia of the bladder wall, a result of obliterative endarteritis, was also present.

All eleven patients completed HBO therapy without suffering any complications and were followed up for a mean period of 17.82 months (range 3 to 34). Mean HBO therapy sessions were 32 (range 27 to 44). Nine men had complete response while the last man and the woman experi- 
enced partial response with marked improvement in their haematuria (grade II). One patient from the complete response group had a recurrence of grade II haematuria at 6 months of follow-up and received 18 additional HBO treatments. All these patients remained with complete response for the rest of the follow-up. The male patient with the partial response received 35 additional treatments and he has not had haematuria since then. The female patient experienced severe haematuria 6 months after the end of HBO therapy and following a full consent she chose cystectomy and urinary diversion for her treatment.

Post-hyberbaric treatment cystoscopy revealed a subjectively normal bladder mucosa in all men, which was confirmed by a histologically normal mucosa in only the 7 patients who had complete response. The male and the female with partial response showed improved but persistent findings of radiation cystitis. Cystectomy specimen revealed a T2G3N0M0 transitional cell carcinoma of the bladder.

Regarding our study endpoints, complete response rate was $81.8 \%$ and partial response rate was $18.2 \%$, giving an overall success rate of primary therapy, as defined in our study, of 100\%. However, as mentioned above, in three patients the first treatment session was not either sufficient or durable giving a $72.7 \%$ rate of durable effect. Interestingly, all 9 patients with complete response received HBO therapy within 6 months of the haematuria onset compared to the two patients with partial response who received HBO therapy at 8 and 10 months from the haematuria onset, respectively $(p<0.018)$. The need for blood transfusion was not correlated to the complete or partial response to HBO $(p=0.491)$. Total radiation dose was not correlated to complete or partial responses to HBO therapy ( $\mathrm{p}=0.259)$. All patients were alive at the end of follow-up.

\section{DISCUSSION}

Bladder complications may occur within 2 months to $>20$ years following completion of pelvic radiotherapy $(1,14)$. Their incidence ranges from 5 to $12 \%$, with haemorrhage presenting in up to $9 \%$ of cases (1). Severe RTOG/EORCT grade III or worse bladder morbidity has been reported at 1\% at 5 years, $1.4 \%$ at 10 years and $2.3 \%$ at 20 years following radiotherapy for cervical cancer (20). Radiation for prostate cancer may lead to moderate or severe haematuria in 3-5\% of cases $(10,12)$.

Traditionally, severe radiation cystitis has been treated in various ways (1). Bladder irrigation almost always constitutes the first-line treatment. Intravesical instillations with alum, silver nitrate, phenol or formalin have been used as a second-line treatment. Several oral and intravenous agents are administered either concomitantly or as a thirdline option. Among them the most commonly applied are aminocaproic acid, traxenamic acid, corticosteroids, estrogens, antibiotics, prostaglandins and sodium pentosanpolysulphate. However, these treatments do not cure the radiation-induced cystitis, nor prevent recurrence of severe haematuria. Moreover, some of them may have serious systematic side effects or may exacerbate bladder fibrosis which was initiated by radiation treatment, leading to a small-capacity bladder (1,2-14). A recent Cochrane Database systematic literature review on non-surgical interventions for late radiation cystitis in patients who have received radical radiotherapy to the pelvis concluded that in the absence of randomized controlled studies it is impossible to set definitive rules for treatment (21). In case of intractable haemorrhage arterial embolization or ligation and/or cystectomy represent definitive treatment, at the cost of increased morbidity.

Pelvic radiotherapy initially causes mucosal edema and inflammation. Telangiectasia, submucosal haemorrhage and interstitial fibrosis follow. Obliterative endarteritis of small blood vessels leads to acute and chronic ischemia of the bladder wall and eventually to smooth muscle fibrosis due to cellular hypoxia (22). Hyperbaria related to HBO therapy increases bladder's tissue oxygen tension (14). Hyperoxia enhances neovascularization and growth of normal tissue $(6,10,15,17)$. Angiogenesis is stimulated by tissue macrophages responding to the steep oxygen gradient (15). Tissue oxygen remains near normal levels for many years following HBO therapy, implying that the angiogenesis is essentially permanent (10). Vasoconstriction and cease of bleeding and improvements of tissue healing and immune function constitute ad- 
ditional beneficial effects of HBO (6,12,15-17). For these reasons, HBO is the only treatment which is believed to reverse the vascular radiation-induced pathophysiology $(15,22)$.

Several series of radiation-induced haemorrhagic cystitis treated with HBO have been reported (2-14). Tables 2 and 3 present indications and results for most of them. The vast majority are retrospective in nature. Endpoints have been set prospectively in only one study (14). These included recurrence of severe haematuria, cystectomy, or death. Forty patients were recruited, all of which had had previous unsuccessful treatments for radiation cystitis. In addition, although a mean transfusion need of 8.2 units per patient was reported, a specific classification system of haematuria morbidity was not used and patients presented with various haematuria grades according to RTOG/EORTC criteria, leading to a diversity of results. Similar to our study, a classification of the severity of haematuria according to transfusion needs was used. However, no statistical analysis was provided and HBO failure was seen only in 3 patients with a mean transfusion need of 25.7 units. Finally, neither the total Gys received nor the time interval elapsed from initiation of HBO therapy to onset of haematuria, were correlated to HBO success rate. Overall, haematuria disappeared completely or improved in 37 patients (92.5\%). With a median follow-up of 23 months, in 9 patients haematuria recurred, representing a recurrence rate of 0.12/year (14). Bladder preservation was achieved in 36 of patients. Based on their results the authors inferred in their conclusion that HBO should not be reserved only for severely affected and conventional therapy-resistant patients.

The retrospective study with the most significant numbers included 62 patients, with a follow-up of 10-120 months (10). Among these patients, all had previous treatments while only 8 (13\%) required pre-hyperbaric oxygen blood transfusion. Of the 57 patients to be evaluated, 49 (86\%) presented with a complete disappearance or a notable improvement of their haematuria. Again, based on their results the authors inferred in their conclusion that early HBO should be considered before repeated instillations of chemicals leave the bladder fibrotic, contracted and noncompliant. Another important retrospective study on 60 patients with intractable to other conservative treatments radiation cystitis, showed an overall $80 \%$ complete or partial response to HBO after 12 months of follow-up (11). Having used similar response criteria to our study, the authors showed that HBO success rate has not been impaired by the modality and timing of pelvic radiotherapy or by previous intravesical treatment. Younger age, higher radiation dose and treatment within 6 months from the onset of haematuria were associated with a favorable HBO outcome. The later, also having been shown retrospectively in other studies (8), was confirmed in our study, in a prospective manner. Tables 2 and 3 summarize data and results for the most of the rest large retrospective studies. All these studies have confirmed the efficacy and safety of HBO therapy.

Our study has several drawbacks. First of all, it is neither randomized nor controlled. However, as cystectomy represents the alternative definitive treatment for radiation cystitis we believe it will be difficult for urologists to randomize patients. Secondly, due to strict inclusion criteria, the number of patients enrolled in our study is small and conclusions should be made with caution. Thirdly, the follow-up in our series is considered short. Although durable results up to 10-years have been published (6), HBO success rate may drop from $73 \%$ to $27 \%$ as follow-up increases from 2.5 years to 5 years (7). As our study is still ongoing, we believe these drawbacks will become weaker upon time.

Taking into consideration the aforementioned drawbacks, current study remains important for several reasons. It is the second prospective study in literature and the first on only haematuria grade IV patients. It is the first study using $\mathrm{HBO}$ as primary therapy to radiation-induced cystitis and the furst in which post-treatment cystoscopic and histologic findings were included to study's endpoints and were documented for all patients. Several conclusions can carefully be drawn from the current study. Primary treatment of severe post-radiation haemorrhagic cystitis with HBO has proved to be effective and safe both for the bladder structure itself and for patients. Initiation of therapy within 6-months of haematuria onset will be of patient's benefit. HBO's success 







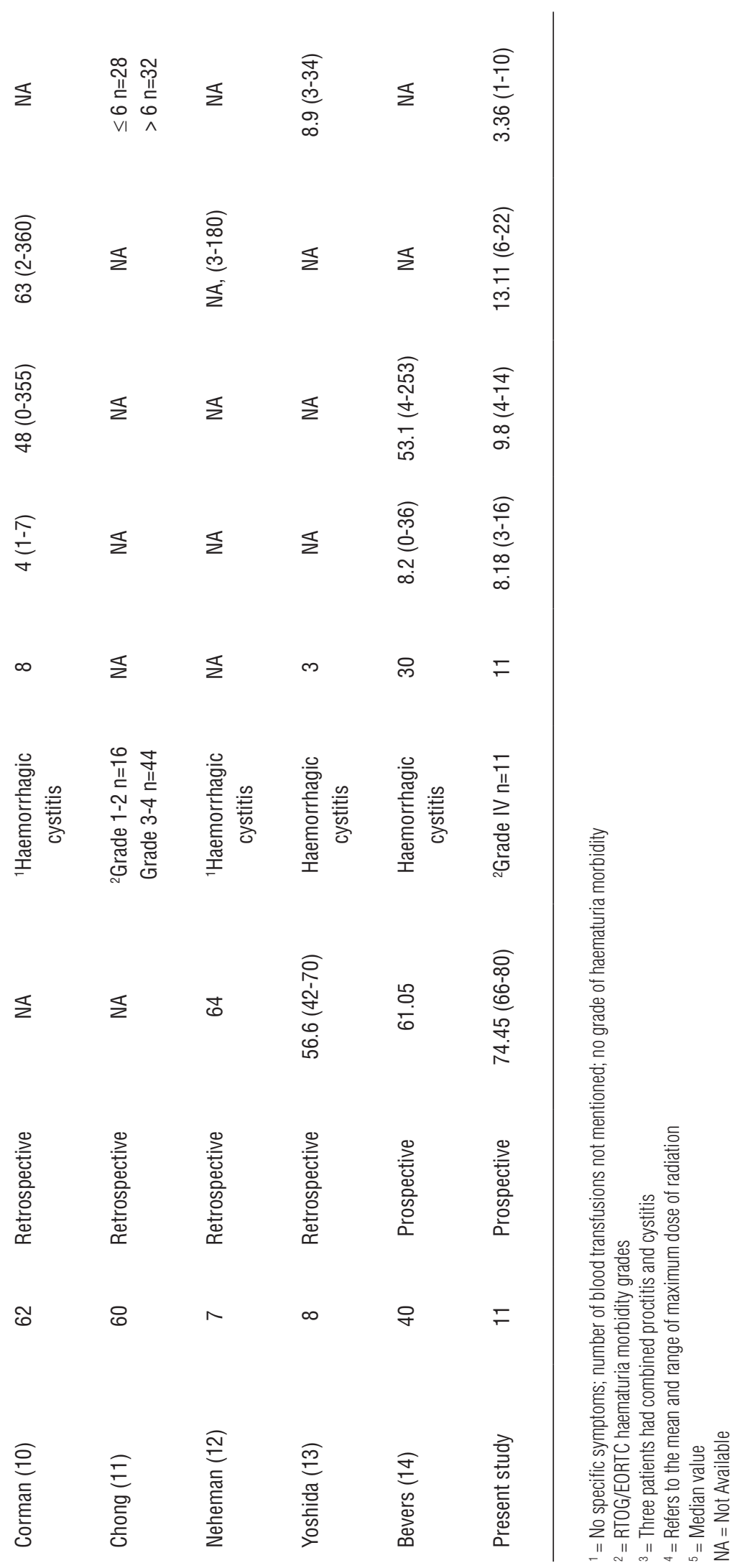









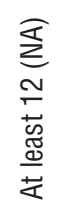

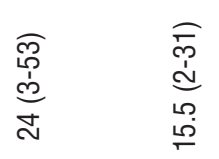

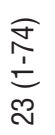

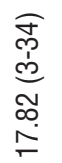

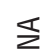

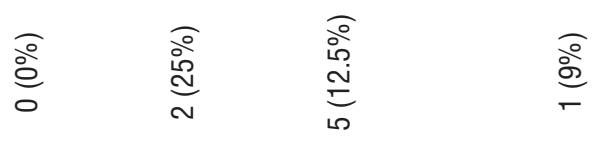

$\Sigma$

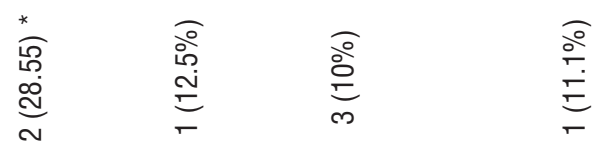

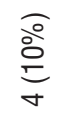

*

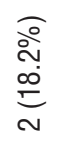

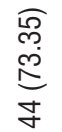

$\stackrel{*}{\circ}$

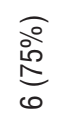

高

$\frac{\widehat{c}}{\infty}$

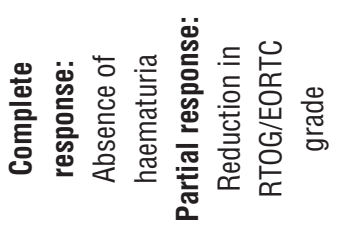

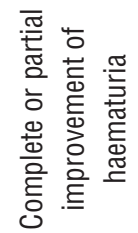

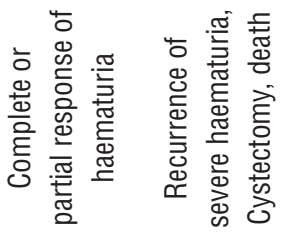

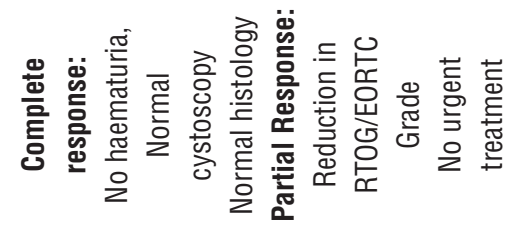

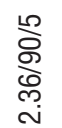

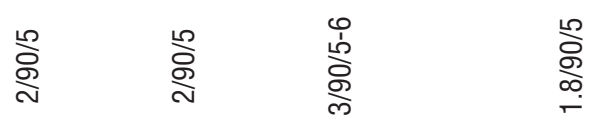

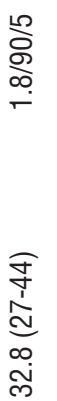

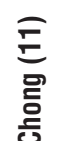

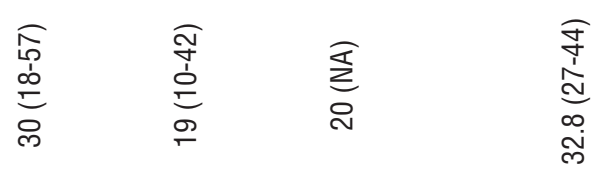

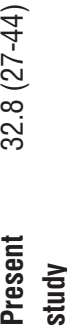

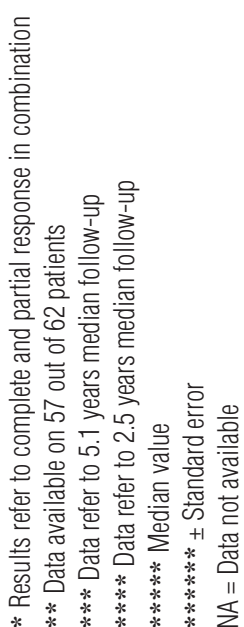


rate is not correlated to the degree of blood transfusion need or radiation dose. Finally, we support the findings of previous series $(2,4)$, indicating that when HBO fails, the urologist should think of other underlying causes such as malignancy.

\section{CONCLUSIONS}

Early primary use of hyperbaric oxygen to treat radiation-induced grade IV haematuria is an effective and safe treatment option. Increase in patient recruitment and longer follow-up is warranted to extract permanent conclusions. Prospective randomized controlled trials will eventually provide information of high-level of evidence.

\section{CONFLICT OF INTEREST}

\section{None declared.}

\section{REFERENCES}

1. Pasquier D, Hoelscher T, Schmutz J, Dische S, Mathieu D, Baumann M, Lartigau E: Hyperbaric oxygen therapy in the treatment of radio-induced lesions in normal tissues: a literature review. Radiother Oncol. 2004; 72: 1-13.

2. Rijkmans BG, Bakker DJ, Dabhoiwala NF, Kurth KH: Successful treatment of radiation cystitis with hyperbaric oxygen. Eur Urol. 1989; 16: 354-6.

3. Nakada T, Yamaguchi T, Sasagawa I, Kubota Y, Suzuki H, Izumiya $\mathrm{K}$ : Successful hyperbaric oxygenation for radiation cystitis due to excessive irradiation to uterus cancer. Eur Urol. 1992; 22: 294-7.

4. Norkool DM, Hampson NB, Gibbons RP, Weissman RM: Hyperbaric oxygen therapy for radiation-induced hemorrhagic cystitis. J Urol. 1993 ; 150: 332-4.

5. Lee HC, Liu CS, Chiao C, Lin SN: Hyperbaric oxygen therapy in hemorrhagic radiation cystitis: a report of 20 cases. Undersea Hyperb Med. 1994; 21: 321-7.

6. Weiss JP, Mattei DM, Neville EC, Hanno PM: Primary treatment of radiation-induced hemorrhagic cystitis with hyperbaric oxygen: 10-year experience. J Urol. 1994; 151: 1514-7.

7. Del Pizzo JJ, Chew BH, Jacobs SC, Sklar GN: Treatment of radiation induced hemorrhagic cystitis with hyperbaric oxygen: long-term followup. J Urol. 1998; 160: 731-3.

8. Mathews R, Rajan N, Josefson L, Camporesi E, Makhuli Z: Hyperbaric oxygen therapy for radiation induced hemorrhagic cystitis. J Urol. 1999; 161: 435-7.

9. Mayer R, Klemen H, Quehenberger F, Sankin O, Mayer E, Hackl $A$, et al.: Hyperbaric oxygen--an effective tool to treat radiation morbidity in prostate cancer. Radiother Oncol. 2001; 61: 151-6.
10. Corman JM, McClure D, Pritchett R, Kozlowski P, Hampson NB: Treatment of radiation induced hemorrhagic cystitis with hyperbaric oxygen. J Urol. 2003; 169: 2200-2.

11. Chong KT, Hampson NB, Corman JM: Early hyperbaric oxygen therapy improves outcome for radiation-induced hemorrhagic cystitis. Urology. 2005; 65: 649-53.

12. Neheman A, Nativ O, Moskovitz B, Melamed $Y$, Stein A: Hyperbaric oxygen therapy for radiation-induced haemorrhagic cystitis. BJU Int. 2005; 96: 107-9.

13. Yoshida T, Kawashima A, Ujike T, Uemura M, Nishimura $K$, Miyoshi S: Hyperbaric oxygen therapy for radiation-induced hemorrhagic cystitis. Int J Urol. 2008; 15: 639-41.

14. Bevers RF, Bakker DJ, Kurth KH: Hyperbaric oxygen treatment for haemorrhagic radiation cystitis. Lancet. 1995; 346: 803-5.

15. Knighton DR, Hunt TK, Scheuenstuhl H, Halliday BJ, Werb Z, Banda MJ. Oxygen tension regulates the expression of angiogenesis factor by macrophages. Science. 1983; 221: 1283-5.

16. Hader JE, Marzella L, Myers RA, Jacobs SC, Naslund MJ: Hyperbaric oxygen treatment for experimental cyclophosphamide-induced hemorrhagic cystitis. J Urol. 1993; 149: 1617-21.

17. Buras J: Basic mechanisms of hyperbaric oxygen in the treatment of ischemia-reperfusion injury. Int Anesthesiol Clin. 2000;38: 91-109.

18. Cox JD, Stetz J, Pajak TF: oxicity criteria of the Radiation Therapy Oncology Group (RTOG) and the European Organization for Research and Treatment of Cancer (EORTC). Int J Radiat Oncol Biol Phys. 1995; 31: 1341-6.

19. Thom TR. Hyberbaric oxygen therapy: a committee report. Bethesda, Maryland: Undersea and Hyberbaric Medicine Society, 1992.

20. Levenback C, Eifel PJ, Burke TW, Morris M, Gershenson DM: Hemorrhagic cystitis following radiotherapy for stage Ib cancer of the cervix. Gynecol Oncol. 1994; 55: 206-10.

21. Denton AS, Clarke NW, Maher EJ: Non-surgical interventions for late radiation cystitis in patients who have received radical radiotherapy to the pelvis. Cochrane Database Syst Rev. 2002; 3: CD001773.

22. Capelli-Schellpfeffer M, Gerber GS: The use of hyperbaric oxygen in urology. J Urol. 1999; 162: 647-54.

Correspondence address:

Athanasios Dellis, MD, PhD 1st Urologic Dept, University of Athens, Aretaieion Hospital 5, Granikou St., 15125 Marousi, Athens, Greece E-mail: aedellis@gmail.com 